\title{
Advanced technologies and applications for Highly-Reliable Cyber Physical System (HRCPS)
}

\author{
Sang Oh Park • Bin Xiao • Victor Leung • \\ Young-Sik Jeong
}

Published online: 22 September 2013

(C) Springer Science+Business Media New York 2013

Advances in information technologies have brought a wide variety of computing environments. In recent years, integrations of computational, networking, and physical elements in different environments tend to increase, so interoperability between heterogeneous computing elements has become a key area of research. A cyber-physical system (CPS) that has gained a lot of attention lately is a tight integration of the system's computational and physical elements. The CPS technology builds on the older discipline of embedded systems, and CPS applications can be found in diverse industry sectors, such as smart home, health care, and transportation. There is a high possibility that a global CPS network that integrates different CPS networks appears in the near future.

CPS is not a new field, but a new type of system which is reflected physical characteristics to embedded system and emphasized importance of controlling between

S.O. Park ( ()

Global Science Experimental Data Hub Center, Korea Institute of Science and Technology

Information, Seoul, Korea

e-mail: pc.sopark@gmail.com

B. Xiao

Department of Computing, Hong Kong Polytechnic University, Hong Kong, Hong Kong e-mail: csbxiao@comp.polyu.edu.hk

V. Leung

Department of Electrical and Computer Engineering, University of British Columbia, Kelowna, Canada

e-mail: vleung@ece.ubc.ca

Y.-S. Jeong (凶)

Department of Multimedia Engineering, Dongguk University, Seoul, Korea

e-mail: ysjeong@dongguk.edu 
systems are connected each other through network. Components of cyber physical system, e.g., controller, sensor, actuator, etc. should transmit the information to cyber space through sensing the environment of real world in real-time, and also they should reflect policy of cyber space to real world in real-time. Therefore, many components of cyber physical system should be available to communicate each other. In addition, cyber physical system consists of controller, sensor, and actuator could reflect the information of cyber space to real world through monitoring a variety of information and controlling actuator in real-time.

So, high reliability technology and application are needed for accurate real-time control in cyber physical system, and data encryption is needed for confidentiality and integrity of data which is transmitted over the network. The aim of this special issue is to invite state of the art in research on highly-reliable cyber physical system (HRCPS). Overall, the special issue will outline the recent trends, major challenges, and perspectives related to highly-reliable cyber physical system.

We have received many manuscripts. Only six manuscripts with high quality were finally selected for this special issue. Each manuscript selected was blindly reviewed by at least three reviewers consisting of guest editors and external reviewers. We present a brief overview of each manuscript in the following.

The first paper entitled "Multi-device application middleware: leveraging the ubiquity of the Web with webinos" by Heiko Desruelle et al. present the webinos platform, a distributed Web runtime platform that leverages the Web for supporting self-adaptive cross-device applications. In order to enable the development of such immersive ubiquitous applications, authors introduce and evaluate the concept of a context-aware federated overlay architecture.

The second paper entitled "Cross-layer optimization for wireless multihop multicast networks" by Wooseong Kim et al. propose a distributed approximation algorithm for the problem. Our numerical results confirm that the benefit of multiuser diversity is prominent in a wireless multihop network with multicast flows.

The third paper entitled "Hierarchical role-based data dissemination in wireless sensor networks" by Chen-Che Huang et al. propose a hierarchical role-based data dissemination (HRDD) protocol in wireless sensor networks. In HRDD, a small number of sensor nodes are assigned to serve as cluster heads and agents to form the data dissemination backbone and mitigate unnecessary query forwarding. In addition, HRDD designs an efficient data delivery mechanism that provides shorter paths to accelerate data delivery as well as reduce the number of data transmissions. An adaptive backbone maintenance mechanism is also introduced for low-energy cluster heads and agents to reduce their load, thereby prolonging the network lifetime.

The fourth paper entitled "Adaptive resource management scheme for monitoring of CPS" by Young-Sik Jeong et al. suggest an adaptive resource management scheme (ARMS) to reduce the loss of sensing information and increase the level of accurate data obtained in the controller manager (CM) among the CPS components. A CPSbased system consists of a number of nodes (sensors and actuators) used to observe or monitor specific areas. ARMS utilizes data about the location and remaining battery capacity of each node to reduce the loss of information due to the irregular lifespans and unexpected breakdowns of resources in the CPS, and to obtain accurate data. Once a broken sensor in the physical world is sensed in the cyber world, the CM 
searches the locations of adjacent alternative nodes within a user-defined range based on the location of the broken node. In this process, an adjacent node search (ANS) algorithm is run to decide on a node (senor or actuator) to replace the broken node, taking into account the remaining battery capacity of candidate nodes. ARMS provides the adaptive resource management function of CPS by sending information on the identity (ID) and destination of the selected node to the controller to move the node to the destination and control the move.

The fifth paper entitled "An efficient strategy of nonuniform sensor deployment in cyber physical systems" Yong-hwan Kim et al. study the sensor deployment pattern problem in cyber physical systems. When designing the sensor deployment pattern, the network lifetime maximization while covering the given area/targets and forwarding sensor data to a sink node is an important issue. In order to prolong the network lifetime by balancing energy depletion across all sensors, authors propose a novel nonuniform sensor distribution strategy. Since sensors located closer to the sink are more involved in data forwarding, sensor densities in different areas should be varied according to the distance to the sink. Based on the nonuniform sensor distribution, authors propose sensor deployment patterns to satisfy the coverage and connectivity requirements and prolong the network lifetime. A numerical computation is performed to validate and compare the effectiveness of the proposed deployment patterns.

The last paper entitled "Random data-aware flash translation layer for NAND flash-based smart devices" by Se Jin Kwon et al. propose the Random Data-Aware Flash Translation Layer (RDA), which enhances the performance and durability of smart devices. RDA improves low space utilization using the state transition. Furthermore, RDA prolongs the durability of the flash memory by spreading out the random data.

Finally, our special thanks go to Professor Hamid R. Arabnia and all editorial staffs for their valuable supports throughout the preparation and publication of this special issue. We would like to thank all authors for their contributions to this special issue. We also extend our thanks to the following external reviewers for their excellent help in reviewing the manuscripts. 